\title{
La contrainte de dose comme outil d'optimisation : mise en ouvre dans le groupe AREVA
}

\author{
V. DECOBERT ${ }^{1}$
}

(Manuscrit reçu le 11 mai 2007, accepté le 5 juillet 2007)

RÉSUMÉ Historiquement chacune des entités nucléaires du groupe AREVA mettait en œuvre séparément la démarche ALARA. Malgré la diversité des métiers pratiqués et la diversité des environnements radiologiques AREVA a choisi de fédérer l'ensemble des entités nucléaires autour d'une politique commune de sûreté nucléaire dont nous rappelons ici les grands principes. Ce processus volontariste, pour lequel l'autorité nucléaire reste incitative, et auquel participent tous les acteurs à chaque niveau de la chaîne hiérarchique, permet d'optimiser les résultats de radioprotection.

ABSTRACT Dose constraint as an optimization tool, implementation in AREVA group.

Historically each nuclear entity of AREVA Company separately implemented ALARA. In spite of the diversity in practices and of the diversity in radiological environment, AREVA chose to federate the whole of the nuclear entities around a common policy of nuclear safety of which we point out the great principles here. The nuclear authority incites this self determining approach in which take part all the actors in each management level. This approach leads to optimize the radioprotection's results.

Keywords: contraints / optimisation / continuous improvment / sustainable development / ALARA

\section{Présentation des métiers d'AREVA}

Le groupe AREVA, qui exerce ses activités sur le marché de l'énergie, regroupe 61000 collaborateurs à travers le monde. La conviction du groupe est que le nucléaire, aux côtés d'autres sources renouvelables, est une solution incontournable pour répondre aux défis énergétiques et environnementaux de la planète. Les métiers du nucléaire y totalisent 38000 personnes, parmi lesquelles près de 20000 font l'objet d'un suivi dosimétrique, et auxquelles s'ajoutent près de 12000 autres surveillées parmi les sous-traitants intervenants sur les sites nucléaires du groupe AREVA.

Ces salariés et sous-traitants exercent leurs activités dans l'ensemble des métiers du cycle du combustible nucléaire et des réacteurs (hors production d'énergie),

\footnotetext{
1 AREVA, 33 rue La Fayette, 75442 Paris Cedex 9, France.
} 
et de la transmission et la distribution d'électricité. Il s'agit de l'extraction et de la purification du minerai d'uranium, de l'enrichissement, de la fabrication de combustibles (combustibles UOx à l'uranium enrichi, combustibles MOx recyclant le plutonium récupéré dans les combustibles usés, autres combustibles), de la conception, de la construction et des services liés à l'exploitation des réacteurs et des usines du cycle, du traitement de combustibles irradiés dans les réacteurs, du transport de combustibles irradiés et de matières radioactives, de l'assainissement et du démantèlement d'installations et de réacteurs.

Les salariés du groupe AREVA concernés par un suivi dosimétrique exercent donc des métiers variés, dans des organisations diversifiées et de tailles très différentes, à travers le monde et donc dans des environnements certes tous très réglementés et contrôlés par une autorité sous tutelle de l'État mais différents. En effet, les limites fixées dans le cadre des obligations réglementaires de l'exploitant sont définies en déclinaison des recommandations de la CIPR, mais se trouvent être plus ou moins contraignantes selon les pays. Par exemple, si la limite réglementaire de dose reste actuellement de $50 \mathrm{mSv} / \mathrm{an}$ aux États-Unis, elle est partout en Europe, en application de la directive européenne 96/29 a minima de $100 \mathrm{mSv}$ sur 5 ans avec un maximum de $50 \mathrm{mSv}$ par an et peut atteindre $20 \mathrm{mSv} / \mathrm{an}$ selon les pays. Une telle diversité, loin d'être un frein, est au contraire un moteur de progrès, car elle permet de définir des objectifs communs volontaristes.

\section{Une politique de développement durable structurée autour de dix grands engagements et mise en œuvre dans une démarche de progrès continu}

AREVA a fait du développement durable la clé de voûte de sa stratégie industrielle, avec la triple ambition d'une croissance rentable, socialement responsable et respectueuse de l'environnement, qui se décline au travers de dix engagements. La prévention et maîtrise des risques technologiques sont l'un de ces dix engagements, pour assurer et maîtriser le plus haut niveau de sûreté et de sécurité dans l'ensemble des activités du groupe afin de préserver la santé des salariés et des populations et de respecter l'environnement (Fig. 1).

La mise en œuvre de ces engagements est fondée sur une démarche volontaire de progrès continu, AREVA Way : des objectifs, communs à toutes les entités, sont fixés dans les documents de politique. Des indicateurs sont définis et une méthode commune de remontée de chaque indicateur et d'auto évaluation des performances est mise en place. Les résultats sont accessibles à chacun. Chaque entité peut ainsi suivre non seulement ses propres progrès, mais également son avancement par rapport à d'autres entités du groupe. 


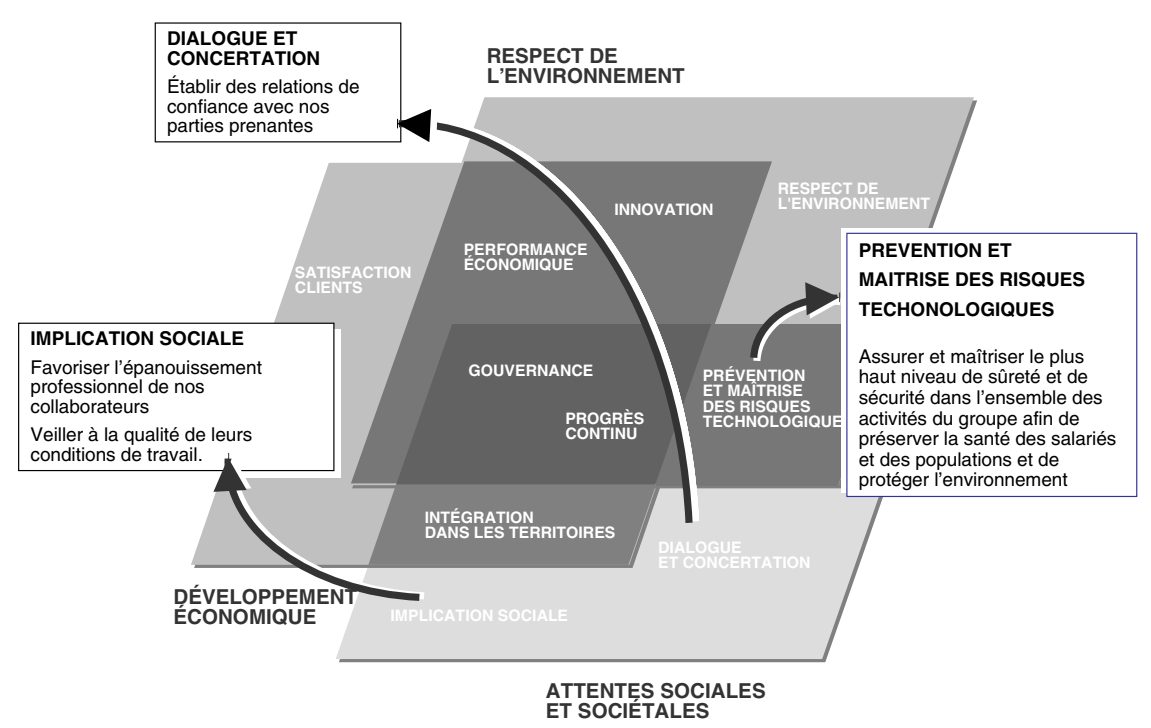

Figure 1 - Les 10 engagements développement durable du groupe AREVA, dont la mise en ouvre est fondée sur une démarche de progrès continu.

The ten AREVA sustainable development commitments based on continuous progress.

\section{Application à la radioprotection}

Historiquement, et bien avant qu'elles ne soient regroupées dans AREVA, chacune des entités nucléaires séparément mettait en œuvre une démarche ALARA. La justification et l'optimisation des pratiques étaient présentes, depuis la conception et au cours de toutes les évolutions des opérations d'exploitation. Par exemple, dans l'aval du cycle, dès la fin des années 70 l'usine de traitement de La Hague a été conçue avec l'objectif qu'aucun travailleur ne dépasse la dose annuelle de $5 \mathrm{mSv}$. C'est sur la base de ce dimensionnement que l'exploitant a ensuite pu faire progresser les performances en matière de dosimétrie.

Cependant, compte tenu de la diversité des métiers pratiqués, de la diversité des environnements radiologiques, de la diversité de culture des entités concernées, les objectifs locaux et les pratiques pouvaient être différents, sans comparaison recherchée entre les différentes entités ; la démarche pouvait se limiter alors à la stricte application des exigences réglementaires sans rechercher une meilleure performance. 
AREVA a choisi de fédérer l'ensemble des entités nucléaires autour d'une politique commune, la Charte de Sûreté Nucléaire, diffusée début 2005. Elle présente des principes d'organisation, des principes d'action et des engagements de transparence.

En matière de radioprotection, la Charte réaffirme l'engagement à limiter dans les installations d'AREVA, à un niveau aussi bas que raisonnablement possible, l'exposition des travailleurs aux rayonnements ionisants, via l'application du principe ALARA et la mise en œuvre d'une politique de progrès continu. Dans ce cadre, AREVA s'engage, dans les pays pourvus d'une législation moins stricte, à ramener à $20 \mathrm{mSv} / \mathrm{individu/an} \mathrm{les} \mathrm{doses} \mathrm{individuelles} \mathrm{maximales} \mathrm{reçues} \mathrm{dans} \mathrm{ses}$ installations par les travailleurs exposés aux rayonnements ionisants. AREVA vise également à respecter cette limite dans le cadre de ses activités de service menées chez ses clients nucléaires. Les entités du groupe en recherchent les modalités d'application en concertation avec ces derniers. Cette approche volontariste, fondamentalement différente du simple respect de limites imposées, modifie radicalement la dynamique de progrès.

Le choix de la valeur objectif de $20 \mathrm{mSv} / \mathrm{an}$ a dû être expliqué en interne : les entités européennes, arrivant déjà pour beaucoup à des performances meilleures, reprochaient à cet objectif d'être peu ambitieux. Quant aux entités dont la réglementation imposait une limite de $50 \mathrm{mSv} / \mathrm{an}$ (USA, Canada et, à l'époque Niger), elles pouvaient craindre de perdre des marchés.

Pour ces entités, la valeur de $20 \mathrm{mSv} / \mathrm{an}$ a été fixée comme un objectif de progrès : c'est à chaque entité opérationnelle de déterminer, en fonction de son marché, en fonction des modalités d'application demandées par les clients notamment, lorsqu'il s'agit d'activités de service, les moyens à mettre en œuvre pour atteindre l'objectif. Dans ce cas de respect d'un objectif groupe commun, c'est l'affichage d'un indicateur commun qui est la contrainte : lorsqu'une entité considère que son marché ne lui permet pas d'atteindre l'objectif, mais que ses dirigeants ont accès à l'ensemble des résultats du groupe, et des progrès des autres entités, cela leur permet d'étalonner leur démarche de progrès par rapport à celle des autres.

Cette démarche n'est pas différente de celle des exploitants de centrales nucléaires, qui ont la chance d'avoir un périmètre de comparaison plus large que les exploitants d'usines du cycle du combustible, lorsqu'ils étalonnent leurs performances en matière de dosimétrie avec celles des autres centrales.

Quant aux entités qui avaient déjà atteint des niveaux inférieurs à $20 \mathrm{mSv} / \mathrm{an}$, en application anticipée de la directive 96/29 et en anticipation de leur réglementation nationale, elles ont continué à se fixer des objectifs de progrès, 


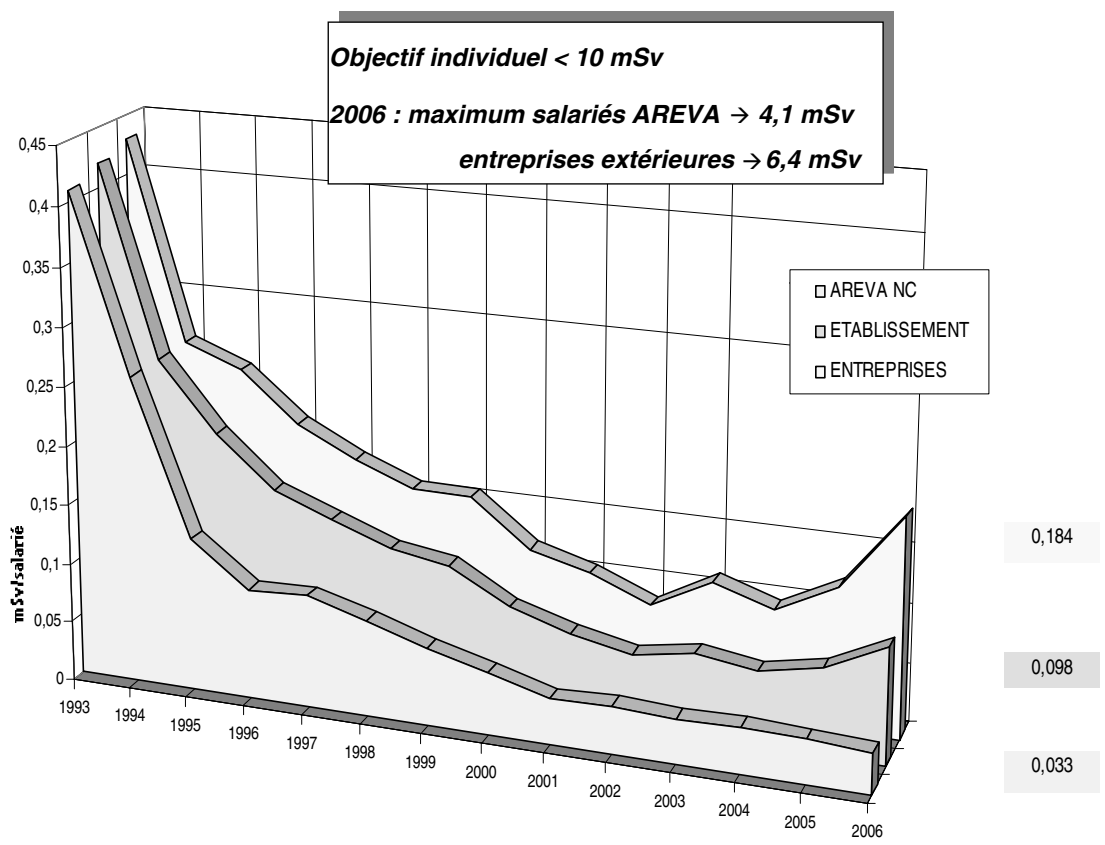

Figure 2 - Évolution des doses moyennes annuelles du personnel surveillé de l'établissement de La Hague (dosimétrie passive).

Evolution of the annual average dose of the monitored workers on the La Hague plant (passive dosimetry).

en ligne avec l'évolution de la conception et du fonctionnement des usines. Là encore, l'affichage du résultat de chaque entité en comparaison de ceux des autres incite à l'amélioration des performances (Fig. 2).

Il faut souligner que cette démarche est déjà dans la culture des exploitants de longue date, jusqu'à tous les niveaux de mise en œuvre opérationnelle : c'est l'application du principe d'optimisation.

Ainsi, chaque établissement fixe, en ligne avec ses activités prévues, des objectifs dosimétriques. En déclinaison, pour un chantier donné, l'objectif de dose est déterminé, lors de l'étude des opérations à mettre en œuvre sur le chantier, en fonction des opérations spécifiques et en fonction du retour d'expérience d'opérations analogues. Dans ce cadre, il faut noter l'importance de la relation entreprise utilisatrice - entreprises extérieures, ces dernières devant respecter non seulement les objectifs imposés par le donneur d'ordre, mais leurs propres valeurs cibles. 
Cet exemple d'optimisation au quotidien est repris à tous les niveaux de l'organisation. Ainsi les indicateurs développement durable, et en particulier ceux relatifs à la radioprotection sont discutés entre les responsables de Business Units et la direction générale lors des revues budgétaires. De nouveaux objectifs de progrès sont fixés, qui sont ensuite déclinés, en tenant compte des spécificités métiers des entités concernées.

Mais il ne faut pas non plus tomber dans l'angélisme. Chacun sait qu'une démarche de progrès, pour être complète, suppose un contrôle de l'effet des dispositions prévues. C'est le rôle des indicateurs de radioprotection. Mais c'est aussi la fonction des contrôles internes tels que prévus dans la Charte Sûreté d'AREVA : contrôles de premier niveau exercés au niveau de chaque directeur d'établissement de façon indépendante des équipes d'exploitation, et contrôle de deuxième niveau par le corps d'inspecteurs de sûreté mis en place au sein de la Direction Sûreté Santé Sécurité.

Ainsi, on le voit bien la démarche d'optimisation repose sur un processus structuré fondé sur l'adoption de ce qui peut être interprété comme des contraintes, mais qui sont bien des objectifs cibles revus régulièrement dans le cadre d'un système de management et de progrès continu. C'est bien cette intégration dans les processus de management de l'entreprise, à tous les niveaux, qui induit une dynamique de progrès durable.

En effet, les individus, à chaque niveau de l'organisation, acceptent mieux la définition d'un objectif, qui a été déterminé avec eux « pour faire mieux que la fois d'avant », qu'une contrainte imposée comme une valeur plafond au-delà de laquelle un dépassement serait assimilé à une faute : c'est la différence entre une démarche participative et une démarche réglementaire ! (voir Fig. 3).

\section{Les résultats}

Alors qu'en 2004, 31 salariés du groupe (et aucun salarié d'entreprise extérieure) présentaient encore une dose maximale supérieure à $20 \mathrm{mSv} /$ individu/an, l'objectif est atteint en 2006 (Figs. 4 et 5).

Il faut cependant souligner que la vigilance qui s'exerce bien entendu sur l'ensemble des activités est surtout focalisée sur les activités de services nucléaires à la demande des électriciens et sur les activités minières. La dose maximale dans les activités de services exercées chez nos clients est très proche de $20 \mathrm{mSv} / \mathrm{individu/an}$. Dans les mines, la limite interne que s'est fixée la Business Unit à $18 \mathrm{mSv} / \mathrm{individu/an}$ est respectée. 


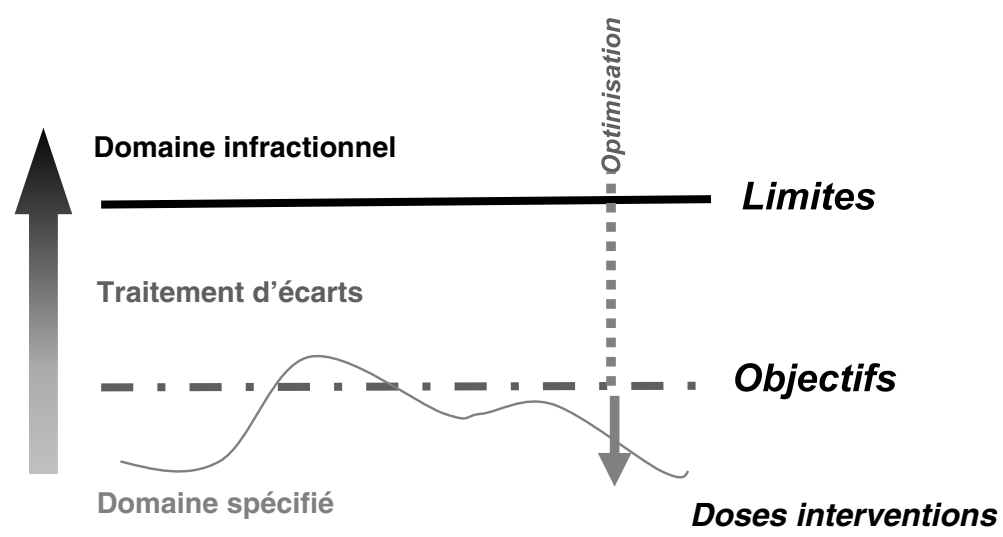

Figure 3 - Les pratiques.

Practices.

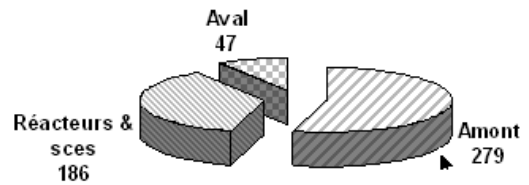

186

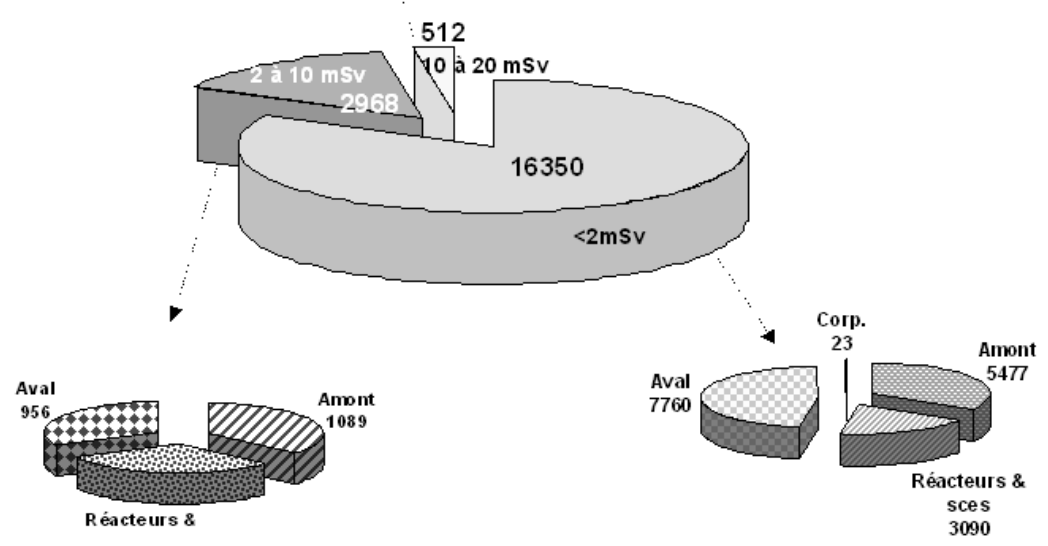

923

Figure 4 - Les résultats radioprotection des travailleurs d'AREVA en 2006. Radiological protection results for AREVA's workers in 2006. 


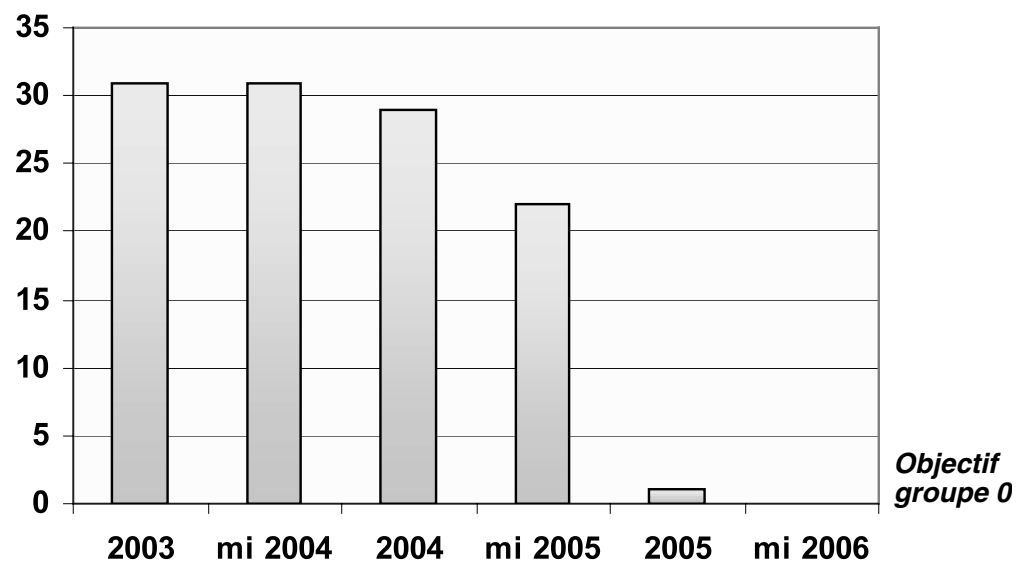

Figure 5 - Évolution du nombre de salariés AREVA ayant une dose supérieure à $20 \mathrm{mSv}$ (les salariés des entreprises extérieures ont une dose inférieure à $20 \mathrm{mSv}$ depuis 2003).

Evolution of the number of AREVA workers having a dose higher than $20 \mathrm{mSv}$ (the subcontractors had doses lower than $20 \mathrm{mSv}$ since 2003).

\section{Établir un champ de contraintes}

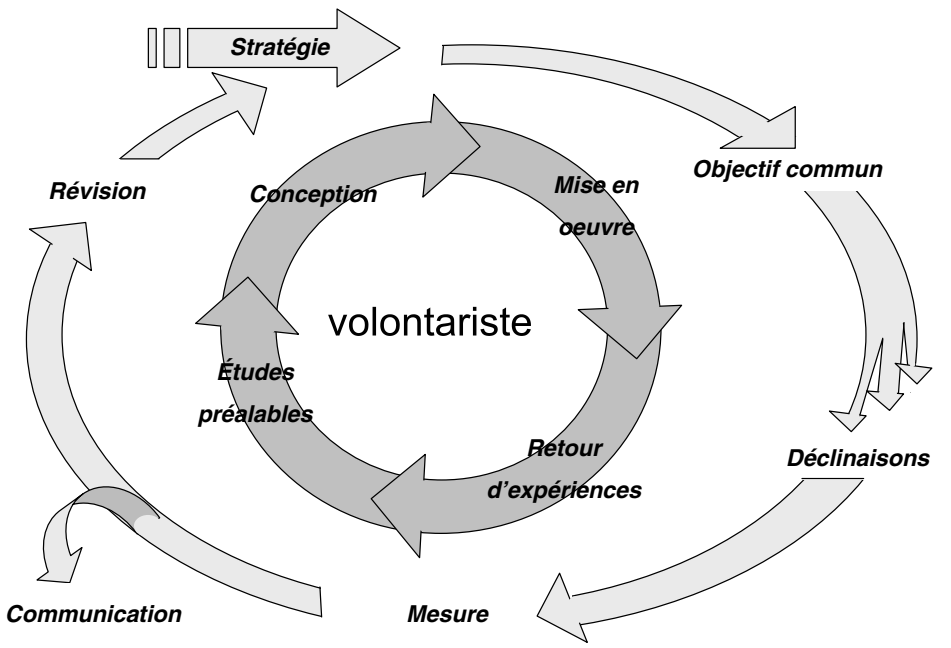

est une garantie de progrès

Figure 6 - Stratégie AREVA.

AREVA strategy. 
Les résultats sont publiés annuellement par AREVA dans son rapport développement durable et présentés sur le site Internet : www.areva.com.

\section{En conclusion}

Au-delà des actions d'optimisation de radioprotection pratiquées sur le terrain, la démarche de progrès continu mise en œuvre par le groupe AREVA constitue un système de contraintes volontaires auquel participent tous les acteurs à chaque niveau de la chaîne hiérarchique. La conception, l'étude préalable des dispositions requises, leur mise en œuvre sur la base du retour d'expérience sont renforcées par la recherche d'un objectif commun fixé au plus haut niveau décisionnel, décliné à chaque niveau de l'entreprise, puis mesuré, affiché, revu et mis en perspective avec la stratégie de l'entreprise (Fig. 6).

Démarche de management participative, ce processus volontariste, pour lequel l'Autorité de sûreté nucléaire reste incitative, est sous la responsabilité de l'exploitant, alors qu'une contrainte plafond telle que le préconisait la CIPR resterait «subie » et risquerait à terme d'être considérée comme infractionnelle.

\section{Pour en savoir plus}

Charte de Sûreté Nucléaire, AREVA (2005).

Rapport annuel de l'état de sûreté des installations nucléaires du groupe AREVA (2005).

Rapport annuel d'activité et de développement durable, AREVA (2006).

Tous ces documents sont disponibles - à télécharger ou commander - sur le site www.areva.com 